\title{
LA DIDÁCTICA DE LA GENÉTICA: REVISIÓN BIBLIOGRÁFICA
}

\author{
BUGALLO RODRíGUEZ, A. \\ Departamento de Didáctica das Ciencias Experimentais. Universidade de Santiago de Compostela. \\ EU Magisterio. R/ Xoán XXIII, s/n. 15704 Santiago de Compostela.
}

\section{SUMMARY}

The aim of this paper is to partially review the historic development and present situation of Genetics Education. Three areas will be examined: 1) Those studies which analyse the significance of the subject, as well as the problems which result from it. 2) How research in this field has been affected by the controversial move to include the subject in the curriculum for the Secondary Education. 3) The growing interest in the studies on Genetics problem solving, and its relationship with the conceptual knowledge.

\section{INTRODUCCIÓN}

La genética es uno de los temas más tratados en la didáctica de la biología debido a su importancia y a que es un área de rápida expansión con importantes implicaciones económicas, éticas y sociales en general (Stewart y Kirk, 1990; Garvin y Stefani, 1993). Está ampliamente reconocida como la base conceptual para la comprensión de la evolución y, por lo tanto, de la propia biología (Smith y Sims, 1992).

Los resultados de los trabajos realizados en enseñanza de la genética han mostrado la necesidad de investigar con mayor profundidad sobre la enseñanza de la biología en general y de la genética en particular.

En los siguientes apartados se revisan en primer lugar, algunos de los trabajos que analizan la dificultad e importancia del tema; en segundo Iugar, los teflejos en la investigación de la controversia creada sobre su inclusión en el currículo de secundaria; y, finalmente, la creciente atención dedicada a los estudios sobre resolyción de problemas de genética y su relación con el conocimiento conceptual.

\section{DIFICULTAD E IMPORTANCIA DE LA GENETICA}

Ein la década de los setenta son escasos los estudios que tratan las creencias de los estudiantes sobre temas de genética. Varios autores (Kargbo et al., 1980) pensaban que quizás esta carencia se debía a la popularidad en aquella época de la «escuela piagetiana», escuela que generalmente sólo empleaba conceptos de física en sus investigaciones para evidenciar el pensamiento operacional y la localización de los sujetos en estadios de desarrollo.

Uno de los trabajos sobre genética en estas fechas es el análisis de las concepciones previas de los estudiantes de secundaria sobre la evolución y la herencia, estudio realizado por Deadman y Kelly (1978). Ambos investigadores indicaron que la inapropiada comprensión de la probabilidad y la ausencia de un concepto simplificado de la herencia mendeliana que actuaban como inclusores eran algunos de los mayores obstáculos para el desarrollo de conceptos más elaborados.

A comienzos de los ochenta se elaboraron dos estudios de gran interés para la didáctica de la genética. El 
primero intentaba determinar cuáles eran los contenidos de biología más difíciles de aprender (Jobstone y Mahmound, 1980), y el segundo analizaba cuáles eran los contenidos considerados más importantes y difíciles por los profesores de ciencias de secundaria (Finley et al., 1982). En ambos trabajos, la genética aparecía en los primeros puestos de importancia y difícultad y, más en concreto, tres de sus áreas: mitosis-meiosis, genética mendeliana y teoría cromosómica.

Tales resultados ayudan a comprender el fuerte incremento de las investigaciones relativas a la genética ocurrido en los años siguientes, tanto sobre las concepciones alternativas de los estudiantes (Albadalejo y Lucas, 1988; Hackling y Treagust, 1984; Kargbo et al., 1980; Kinnear, 1983 y Mahadeva y Randerson, 1982), como sobre las dificultades en la resolución de problemas de genética (Longden, 1982; Radford y Bird-Stewart, 1982; Tolman, 1982).

Se ha investigado en qué medida estas concepciones alternativas de los estudiantes eran debidas a los contenidos de los libros de texto (Cho et al., 1985, Tolman, 1982), y se ha mostrado la coincidencia de tales ideas en estudiantes de países lejanos entre sí, tanto geográfica como culturalmente (Hackling y Treagust, 1984, Stewart et al., 1990). Varios trabajos orientados a explorar qué temas de genética eran particularmente difíciles han avanzado un paso más y han apuntado ideas para reducir esta dificultad (Gené, 1991; Jiménez, 1991; Radford y Bird-Stewart, 1982 y Smith, 1991).

Algunas de las principales fuentes de concepciones alternativas y de dificultades para el aprendizaje de la genética identificadas en estas investigaciones son:

- El uso de la terminología

1. La semejanza superficial entre los procesos de división celular, mitosis y meiosis provoca confusión entre los términos y oculta lo significativo, que son las diferencias entre ambos procesos (Radford y Bird-Stewart, 1982; Smith, 1991).

2. En los libros de texto se usan de forma incorrecta y ambigua ciertos conceptos genéticos, por ejemplo, gen y alelo se emplean indistintamente, sin establecer su significado correcto (Cho et al., 1985).

3. La confusión provocada por el uso dado a diversos términos genéticos en el lenguaje coloquial, como es el caso de "mutation/mutación» (Albadalejo y Lucas, 1988) o de «ligado" (Kinnear, 1991).

- Las relaciones entre conceptos:

1. Una de las mayores fuentes de problemas es el tema de la meiosis. Cuando se enseña meiosis es esencial relacionarla con la fertilización, los ciclos de vida y la alternancia de generaciones haploides y diploides (Radford y Bird-Stewart, 1982; Smith, 1991).

2. El no establecer previamente una serie de relaciones importantes (Cho et al., 1985) como:
- separación cromosómica - replicación de DNA;

- par alélico - expresión del rasgo;

- separación cromosómica - transmisión del rasgo.

3. La falta de claridad en las relaciones especificicas entre los siguientes conceptos básicos: alelo, gen, DNA, cromosoma, rasgo, gameto, zigoto (Cho et al., 1985).

- Resolución de problemas

1. A diferencia de otras áreas de la biología, la enseñanza de la genética requiere un nivel mayor de matemáticas y de capacidad analítica, sobre todo para la resolución de problemas (Longden, 1982; Radford y Bird-Stewart, 1982).

2. Los estudiantes pueden Ilegar a resolver con éxito los problemas, pero sin encajar el algoritmo en el contexto del proceso genético (Stewart, 1983; Thomson y Stewart, 1985).

\section{- El trabajo práctico}

Una fuente adicional de dificultades viene derivada del trabajo práctico en genética. Los experimentos clásicos necesitan semanas o meses para realizarse, lo que resulta incompatible con el ritmo escolar (Beals, 1995; Radford y Bird-Stewart, 1982).

\section{INFLUENCIA DEL DEBATE CURRICULAR EN LA INVESTIGACION EN DIDACTICA DE LA GENÉTICA}

En Gran Bretaña antes đe 1960 los temas đe genética no estaban incluidos en los programas para menores de 16 años. Pero los proyectos curriculares británicos de los sesenta pasaron a tratar herencia y evolución como conceptos centrales para la comprensión de la biología, $\mathrm{y}$, por lo tanto, como parte de la educación general antes de los 16, llegando a aparecer en el proyecto para los niveles de 5 a 13 años. De forma semejante se incluyeron estos temas en nuevos cursos de otros países, como es el caso de Estados Unidos.

La introducción de temas de genética en el currículo puso de manifiesto la diversidad de opiniones existente sobre si era apropiado enseñar los temas de herencia y evolución a estudiantes de 16 años o menores. La aparente contradicción entre la importancia del tema y su dificultad ha derivado en una controversia que ha llegado hasta la actualidad: se discute la conveniencia de incluir temas de genética y evolución en el recién reformado currículo de ciencias de los 12 a los 16 años en España, o toda la genética clásica en el currículo americano para enseñanza secundaria (Gipson et al., 1989).

La polémica ha impulsado nuevas investigaciones sobre la comprensión del tema, con una orientación clara en respuesta a las implicaciones que sus resultados tienen para los diseñadores del currículo de ciencias. Se han 
aportando soluciones como, por ejemplo, nuevas secuencias didácticas (Tolman, 1982) o la lista de conceptos y proposiciones necesarias para comprender la genética (Hackling y Treagust, 1984), y se ha indicado la posible existencia de una estrecha conexión entre los conocimientos de genética y las dificultades en el aprendizaje de la evolución (Jiménez, 1992).

La controversia sobre genética posiblemente está relacionada con las dos tradiciones que han dominado la investigación sobre su aprendizaje.

- En la base de los argumentos contra el mantenimiento de la genética en el currículo, se encuentran los estudios de algunos investigadores piagetianos sobre las demandas cognitivas para la resolución de los problemas de esta materia. Especialistas como Lawson (Lawson, 1983; Mitchell y Lawson, 1988) han argumentado que el estadio de desarrollo cognitivo de un estudiante puede utilizarse para explicar su éxito o su fracaso en un contenido particular. Han calificado el contenido de esta disciplina como «hipotético», y tales conceptos necesariamente formales son difíciles de comprender por las y los estudiantes que se encuentian en el estadio de operaciones concretas.

De sus estudios se deriva que la carencia de đestrezas apropiadas de razonamiento hipotético-deductivo (por ejemplo, capacidad de razonamiento combinatorio, probabilístico y proporcional) serían ta principal fuente de dificultad, no sólo a la hora de resolver problemas de genética, sino tambiến para interpretar sus textos. Acusan a autores como Hafner y Stewart de ser incapaces de reconocer el uso de patrones de razonamiento en la resolución de problemas de genética, y de no comprender la implicación en el aprendizaje de variables tales como la capacidad mental (Lawson, 1989).

Pero no todos los análisis según el modelo piagetiano han llegado a la misma conclusión. Con anterioridad, Haley y Good (1976) habian indicado que, en contra de lo sugerido por el modelo tradicional piagetiano de desarrollo cognitivo, la mayoría de los estudiantes universitarios de primer curso continuaban careciendo de modelos de razonamiento formal. Según Walker, Hendrix y Mertens (1980) Ia permanencia en el nivel de pensamiento operacional concreto es debida a la falta de experiencias que refuercen el razonamiento desde el punto de vista formal. Indican además que las operaciones formales piagetianas de lógica combinatoria, lógica proposicional y razonamiento hipotético deductivo están relacionadas con el método escolástico utilizado en el curso de genética introductorio a la universidad. Por lo tanto, consideran necesario desarrollar estrategias didácticas que faciliten el desarrollo cognitivo de los estudiantes cara a las formas de pensamiento de operaciones formales. Proponen una secuencia didactica diseñada para mejorar la capacidad de los estudiantes a la bora de aplicar tales modelos de pensamiento a problemas de genética mendeliana.

En la misma linea, Hackling y Treagust (1984) afirman que consideran posible el mantenimiento de la genética en el currículo para menores de 16 años si se modifican los ntateriales curriculares. Aunque están de acuerdo en que los estudiantes de 15 años están limitados al pensamiento operacional concreto y reafirman la naturaleza abstracta de los conceptos implicados, ambos autores opinan que los estudiantes son capaces de comprender el fenómeno de la herencia cuando se desarrolla desde experiencias concretas y familiares, y cuando los profesores ilustran las características no perceptibles de estos conceptos.

- Varios autores partidarios de la inclusión de la genética en el currículo basan sus investigaciones en los trabajos de Ausubel. Parten de que el conocimiento previo conceptual relevante es el factor más importante tanto en el aprendizaje de contenidos científicos como en el uso de ese conocimiento para resolver problemas.

En contra de la opinión de otros investigadores de la época (Shayer, 1974), Deadman y Kelly (1978) eran favorables a la inclusión đe la genética en el currículo para menores de 16 años. Apuntaban dos razones: por un lado, la importancia social y científica del tema y, por otro, que consideraban factible descubrir métodos apropiados para presentarlo en ese nivel educativo. Tales métodos se basaban en el desarrollo curricular de alumno a curriculo, es decir, un desarrollo que tuviera en cuenta las ideas que los estudiantes traen a la escuela. Partiendo de la investigación previa de la comprensión que los estudiantes tenían del tema, se abría un proceso de elaboración gradual, donđe se combinaba desarrollo, investigación y enseñanza, y se exploraba directamente en clase, observando cómo se producía el aumento de la comprensión.

Otros argumentos presentados a favor de la inclusión proceden de estudios recientes en los que se destaca la importancia del conocimiento específico del tema y de las destrezas a la hora de comprender y resolver problemas de genética (Smith, 1991; Stewart, 1982a). Así Stewart (1982a) explica que los estudiantes no presentan carencia de operaciones mentales para generar combinación, pero carecen de conocimientos para relacionar, por ejemplo, los porcentajes con la segregación de cromosomas. Y, junto a Hafner (Stewart y Hafner, 1989), critica a Mitchell y Lawson por utilizar instrumentos que no sirven para valorar la organización del conocimiento conceptual en los estudiantes ni su retación con el conocimiento procedimental.

Smith y Sims (1992) creen haber eliminado el argumento principal contra la inclusión de la genética, pues opinan que el significado del têrmino «hipotético» en este contexto no está claro, y que es indefendible una interpretación estricta de los estadios piagetianos. Consideran que, aun cuando numerosos conceptos genéticos son en gran medida formales, los estudiantes en el nivel concreto operativo poseen muchas de las destrezas cognitivas requeridas para resolver los problemas más típicos de la genética clásica. Afirman que las técnicas didácticas son capaces de impulsar en todos los estudiantes de secundaria la compresión de los conceptos genéticos. Ambos autores concluyen que una enseñanza 
efectiva que tenga en cuenta los niveles cognitivos de los estudiantes demandará cambios radicales en las técnicas educativas, una reducción sustancial del númeto de conceptos formales introducidos y un incremento en la cantidad de tiempo que se debe emplear para dar sentido a estos conceptos.

Un último factor a favor de la inclusión de la genética en el currículo es el consenso creciente entre los especialistas en didáctica de ciencias sobre la necesidad de que los estudiantes y el público en general comprendan mejor los conceptos genéticos básicos. La importancia de comprender este tema parece cada vez mayor a medida que las sociedades encuentran cuestiones éticas, sociológicas y ecológicas que tienen sus raíces en la investigación genética actual. Según Thomson y Stewart (1985), es más probable que se tomen decisiones racionales sobre temas como armas genéticas, ingenietía genética, preservación de la diversidad de la reserva genética, y productos mutagénicos elaborados tecnológicamente si los individuos que las toman comprenden la genética básica que si no la comprenden.

\section{LA RELACIÓN ENTRE CONOCIMIENTO CONCEPTUAL Y RESOLUCIÓN DE PROBLEMAS}

Como se ha señalado anteriormente, estudios recientes han sugerido que los investigadores no sólo deberían enfocar su atención sobre el contenido de la instrucción como una variable importante de la investigación, sino también darle una importancia creciente a la resolución de problemas.

Durante la última década, los mayores esfuerzos de investigación en didáctica de la genética se han centrađo precisamente en la relación entre el conocimiento conceptual y las estrategias de resolución de problemas. En primer lugar, se ha intentado describir la organización del conocimiento y la resolución de problemas por estudiantes y especialistas (Hackling y Lawrence, 1988; Simmons y Lunnetta, 1993; Smith, 1988; Smith, 1992; Smith y Good, 1984), y, en segundo lugar, se ha buscado desarrollar un esquema de enseñanza en un ambiente de resolución de problemas, especialmente por parte del equipo dirigido por James Stewart en la Universidad de Wisconsin-Madison (Slack y Stewart, 1990; Stewart, 1983; Stewart y Kirk, 1990).

Uno de los primeros resuitados de tales investigaciones (Stewart, 1983) indica que las respuestas correctas a los problemas no significan necesariamente que se esté usando un conocimiento conceptual apropiado para justificar los pasos de resolución del problema. Los estudiantes pueden utilizar algoritmos, por ejemplo, el cuadradode Punnet, sin comprender el conocimiento conceptual que subyace en ellos. Stewart (1982 a y b) considera que existe «resolución significativa» de los problemas cuando los estudiantes pueden explicar en términos de gené- tica por qué realizaron cada paso. Partiendo de este argumento elabora una clasificación de problemas diferenciando aquéllos que implican razonamiento «causaefecto", que son la mayoría de los encontrados en los libros de texto y que generalmente llevan a soluciones algorítmicas, frente a los que implican razonamiento «efecto-causa" (Stewart, 1988).

La atención especial prestada a la resolución de este último tipo de problemas está basada en la nueva orientación didáctica cara a «la ciencia en acción». Son problemas que permiten la elaboración de estrategias que reflejan todo el proceso de investigación que la resolución đe un auténtico «problema» debe entrañar (Gil y Martínez, 1983; Stewart y Hafner, 1991). Se trata de evitar que los datos sean tomados como punto de partida de la resolución, Io que se convertiría entonces en la simple aplicación de un algoritmo (Moll y Allen, 1987 y Nackhelh y Mitchell, 1993). Los estudiantes trabajan en "grupos de investigación" para diagnosticar problemas, construir modelos con el fin de explicar los fenómenos, y defender y criticar los modelos (Johnson y Stewart, 1990).

Se están investigando una amplia serie de tales estrategias didácticas (Balachadran et al., 1993; Hafner, 1992; Jiménez, 1990; Johnson y Stewart, 1990), incluyendo el uso de la analogía y de los organizadores gráficos avanzados, que requieren que los estudiantes construyan el problema, estrategias de trabajo en grupo, y la utilización de destrezas cognitivas en la enseñanza diaria.

Un ejemplo de estas estrategias es el currículo único "Gregor Mendel», elaborado por Johnson y Stewart (1990), con el que los estudiantes experimentan aspectos de la «naturaleza de la ciencia», tal y como se estudian en genética clásica. En este currículo, «el propio Mendel» presenta su modelo conceptual de dominancia simple e implica a los estudiantes en una reproducción de su famoso experimento. Los estudiantes Ieen su artículo y prueban st modelo usando un programa informático de simulación. Luego, usando el simulador, se les presenta una población-campo que expresa datos anómalos. Trabajando en grupos de investigación, hacen experimentos genéticos y construyen modelos conceptuales para explicar sus datos y hacer predicciones sobre cómo se generan. Los problemas requiren que los estudiantes trabajen desde los efectos (fenotipo) a las causas (modelos de genotipo), tal y como hacen los profesionales de la genética. Además de que el profesor presente el contenido, Ios estudiantes construyen sus modelos conceptuales y se los presentan a otros.

Tales situaciones-problema precisan que los estudiantes trabajen dentro del contexto de descubrimiento-revisión de los modelos existentes para encajar nuevos datos (Hafner, 1992), implicándolos más íntimamente en la resolución. Resuelven problemas con un nuevo objetivo, la elaboración de teorías. La utilización en la enseñanza de estos modelos de ejecución ayudará a que los estudiantes desarrollen conexiones explícitas entre conocimiento conceptual y resolución de problemas (Slack y Stewatt, 1990). 
Respecto al tema de la organización del conocimiento, varias de estas investigaciones que incorporan estrategias dinámicas de investigación, usando como medio un ordenador, intentan examinar la información sobre el proceso cognitivo de los estudiantes. De la natureza exploratoria de estos estudios surgen muchas cuestiones de interés y se generan hipótesis sobre la naturaleza de la resolución de los problemas y el desarrotlo de los conceptos en genética. Simmons y Lunetta (1993) examina* ron los comportamientos de resolución de problemas y los conceptos genéticos empleados por expertos y novatos durante su interacción con el programa de simulación CATLAB. Los sujetos realizaban investigaciones, cuyos resultados eran utilizados por ambos autores para deducir modelos generales de comportamiento en resolución de problemas y organizadores conceptuales genéticos. De esta forma, Simmons y Lunetta pudieron analizar y describir fas características específicas o comunes de los sujetos.

De este modo la investigación sobre cognición puede arrojar nueva luz a la influencia del conocimiento previo de la persona que aprende en la adquisición del nuevo conocimiento. Se está proporcionando un importante enlace entre el conocimiento disciplinar, en este caso de la genética, y cómo se aprende y se usa ese conocimiento (Smith, 1992; Stewart y Kirk, 1990). La adquisición de un concepto científico parece implicar la capacidad de saber usarlo en sus relaciones con otros conceptos, dentro de una estructura conceptual. Por lo tanto, el conocimiento del concepto científico es más procedimental (Hasweh, 1986) o funcional (Jiménez, 1992) que declarativo, es decir, implica la capacidad de transferirlo a un contexto diferente. Según Smith (1992) la educación debería diseñarse con el fin de proporcionar a los estudiantes la capacidad de reestructurar su organización del conocimiento para promover cambios de uso. Tal capacidad sería muy valorable en aquellos momentos en los que se requiere el uso en el mundo profesional del conocimiento ganado en la educación formal, así como para la toma de decisiones de carácter social.

Algunos de los últimos trabajos sobre resolución de problemas estudian un nuevo factor determinante para el aprendizaje significativo de la genética, aparte del ya señalado conocimiento previo; este factor es la uorientación de cara al aprendizaje significativon. Estos trabajos se han centrado en descubrir cómo "orientan su aprendizaje» los estudiantes, es decir, en qué medida los estudiantes afrontan el aprendizaje de una materia con la intención de comprender significativamente la ideas y las relaciones implicadas. Cavallo y Schafer (1994) clasifican a los estudiantes según su método para aprender nuevos conceptos, en:

- los que aprenden de forma rutinaria (responden dando definiciones establecidas y no pueden extrapolar sus ideas);

- los que aprenden significativamente (responden a los nuevos problemas cuestionándose a sí mismos y relacionando y elaborando ideas);

- y los que están a medio camino.

De la investigación llevada a cabo, estos autores deducen que la «orientación de los estudiantes de cara al aprendizaje significativo» puede ser importante, quizás tanto o más que la aptitud y motivación, para llegar a una comprensión significativa e interrelacionada de la ciencia. Consideran que, además de la orientación, también el método didáctico utilizado, «receptivo» o "generativo», influirá en los resultados de comprensión.

En resumen, la nueva tradición de investigación que surge en la didáctica de la genética bajo el amparo de la investigación sobre cognición orientada por la corriente constructivista, trata explícitamente la relacion entre el conocimiento conceptual y las estrategias de resolución de problemas. Esta relación se investiga a través del análisis de la «orientación de aprendizaje» y de los modelos empleados por los estudiantes, los cuales incluirán los pasos procedimentales, utilizados para resolver problemas, y el conocimiento conceptual de genética, usado para justificar la solución.

Tal investigación ha producido, en los últimos catorce años, informes descriptivos del comportamiento de los estudiantes y de los profesionales cuando resuelven problemas y de su «orientación» cuando aprenden genética. A partir de los informes se han hecho sugerencias para modificar la enseñanza, encaminadas a mejorar la capacidad de los estudiantes para resolver problemas y a facilitar la comprensión significativa de nuevos conceptos de genética.

\section{AGRADECIMIENTOS}

Agradezco a la Xunta de Galicia la financiación del proyecto XUGA 22701B93, del cual es parte este estudio. 


\section{REFERENCIAS BIBLIOGRÁFICAS}

ALBADALEJO, C. y LUCAS, A. (1988). Pupils' meanings for "mutation". Journal of Biological Education, Vol. 22 (3), pp. 215-219.

BALACHADRAN, J., HANG, Y., HENAO, S., MORALES, $X$., OLOWOPOPO, S., PATIÑO, M., SERATE, J. Y VELEZ, S. (1993). How is eye color inherited? Bioquest Notes, Vol. 4 (1), pp. 8-16.

BEALS, J.K. (1995). Creative genetics. A lab for all seasons. Journal of College Science Teaching, Vol. 24 (3), pp. 183-185.

CAVALLO, A.M.L. y SCHAFER, L.E. (1994). Relationships between students' meaningful fearning orientation and their understanding of genetic topics. Journal of Research in Science Teaching, Vol. 31 (4), pp. 393-418.

CHO, H.M., KAHLE, J.B.y NORDLAND, F.H. (1985). An investigation of high school biology textbooks as sources of misconceptions and difficulties in genetics and some suggestions for teaching genetics, Science Education, Vol. $69(5)$, pp. 707-719.

DEADMAN, J.A. y KELLY, P.J. (1978). What do secondary schools boys understand about evolution and heredity before they are taught the topics? Journal of Biological Education, Vol. 12 (1), pp. 7-15.

FINLEY, F., STEWART, J.y YARROCH, W. (1982). Teacher's perceptions of important and difficult science content: The report of a survey. Science Education, Vol. 66, pp. $531-538$.

GARVIN, W. y STEFANI, L. (1993). Genetics-genetic disorder and diagnosis: a role-play exercise. Journal of Biological Education, Vol. 27 (1), pp. 51-57.

GENÉ, A. (1991). Cambio conceptual y metodologico en la enseñanza y el aprendizaje de la evolución de los seres vivos. Un ejemplo concreto. Enseñanza de las Ciencias, Vol. 9(1), pp. 22-27.

GIL, D. y MARTíNeZ, J. (1987). Los Programas-Guía de Actividades: una concreción del modelo constructivista de aprendizaje de las ciencias. Investigación en la Escuela, Vol. 3, pp. 3-12.

GIPSON, M.H., ABRAHAM, M.R. y RENNER, J.W. (1989). Relationships between formal-operational thought and conceptual difficulties in genetics problem solving. Journal of Research in Science Teaching, Vol. 26, pp. 811-821.

HACKLING, M.W. y LAWRENCE, J.A. (1988). Expert and novice solutions of genetic pedigree problems. Journal of Research in Science Teaching, Vol. 25 (7), pp. 531-546.

HACKLING, M.W. y TREAGUST, D. (1984). Research data necessary for meaningful review of grade ten high school genetics curricula. Journal of Research in Science Teaching, Vol. 21 (2), pp. 197-209.

HAFNER, R.S. (1992). Constructing explanatory models for anomalous genetic phenomena: Ploblem solving in the «Context of Discovery", NARST.

HAFNER, R.S. y STEWART, J.(1989). A comment on predicting genetics achievement in nonmajors college biology.Journal of Research in Science Teaching, Vol. 26 (6), pp. 55.

HALEY, S.B. y GOOD, R.G. (1976). Concrete and formal operational thought: implications for introductory college biology. The American Biology Teacher, Vol. 38 (7), pp. 407 .
HASWEH, M. (1986). Toward an explanation of conceptual change. European Journal of Science Education, Vol. 8 (3), Pp. 229-249.

JMÉNEZ ALEIXANDRE, M.P. (1990). Xogos de simulación e modelos de Xenética. Ciencias Revista de Enseñanza, Vol. 9 (3), pp. 248-256.

JKMÉNEZ ALEIXANDRE, M.P. (1991). Cambiando las ideas sobre el cambio biológico. Enseñanza de las Ciencias, Vol. 9-10, pp. 114-122.

JMMÉNEZALEIXANDRE, M.P. (1992). Thinking about theories or thinking with theories? A classroom study with Natural Selection. International Journal of Science Education, Vol. 14 (1), pp. $51-61$.

JOHNSON, S.K. y STEWART, J. (1990). Using philosophy of science in curricula development: an example from high school genetics. International Journal of Science Education, Vol. 12 (3), pp. 297-307.

JOHNSTONE, A.H. y MAHMOUD, N.A. (1980). Isolating topics of high perceived difficulty in school biology. Journal of Biological Education, Vol. 14 (2), pp. 163-166.

KARGBO, D.B., HOBBS, E.D.y ERICKSON, G.L. (1980). Children's beliefs about inherited characteristics. Journal of Biological Education, Vol. 14 (2), pp. 137-146.

KINNEAR, J.F. (1991). Using a historical perspective to enrich the teaching of linkage in Genetics. Science Education, Vol. 75 (1), pp. 69-85.

LAWSON, A.E. (1983). Predicting science achievement: The role of developmental level, disembedding ability, mental capacity, prior knowledge, and beliefs. Journal of Research in Science Teaching, Vol. 20 (2), pp. 117-129.

LAWSON, A.E. (1989). A reply to Hafner and Stewart's comments on «Predicting genetics achievement in nonmajors college biology". Journal of Research in Science Teaching, Vol. $26(6)$, pp. 555-556.

LONGDEN, B. (1982). Genetics: are there inherent learning difficulties? Journal of Biological Education, Vol. 16, pp. $137-146$.

MAHADEVA, M.N. y RANDERSON, S. (1982). Mutation: Mumbo jumbo. Science Teaching, Vol. 49, pp. 135-140.

MITCHELL, A. y LAWSON, A.E. (1988). Predicting Genetics achievement in nonmajors college biology.Journal of Research in Science Teaching, Vol. 25 (1), pp. 551-553.

MOLL, M.B. y ALLEN, R.D. (1987). Student difficulties with Mendelian genetics problems. The American Biology Teacher, Vol. 49 (4), pp. 229-233.

NACKHLEH, M.B. y MITCHELL, R.C. (1993). Concept learning versus problem solving. Journal of Chemical Education, Vol. 70 (3), pp. 190-192.

RADFORD, A. y BIRD-STEWART, J.A. (1982). Teaching genetics in schools. Journal of Biological Education, Vol. 16 (3), pp. 177-180.

SHAYER, M. (1974). Conceptual demand in the O-level Nuffield Biology Course. School Science Review, Vol. 56 (195), pp. 381-388.

SIMMONS, P.E. y LUNETTA, V.L. (1993). Problem-solving during genetics computer simulation: Beyond the expert/ novice dichotomy. Journal of Research in Science Teaching, Vol. $30(2)$, pp. 153-173. 
SLACK, S.J. y STEWART, J. (1990). High school students' problem -solving performance on realistic genetics problems. Journal of Research in Science Teaching, Vol. 27 (1), pp. 55-67.

SMITH, M.U. (1988). Successful and unsuccessful problem solving in classical genetic pedigrees. Journal of Research in Science Teaching, Vol. 25 (6), pp. 411-433.

SMITH, M.U. (1991). Toward a unified theory of problem solving: Views from the content domains. Erlbaum: Hillsdale.

SMITH, M.U.(1992). Expertise and the organization of knowledge: Unexpected differences among Genetic counselors, Faculty and students on problem categorization tasks. Journal of Research in Science Teaching, Vol. 29 (2), pp. 179-205.

SMITH, M.U. y GOOD, R. (1984). Problem solving and classical genetics: Successful versus unsuccessful performance. Journal of Research in Science Teaching, Vol. 21 (9), pp. 895-912.

SMITH, M.U. y SIMS, O.S. (1992). Cognitive development, genetics problem solving, and genetics instruction: A critical review. Journal of Research in Science Teaching, Vol. 29 (7), pp. 701-713.

STEWART, J. (1982a). Difficulties experienced by high school students when leaming basic Mendelian gentetios. The American Biology Teacher, Vol. 44 (2), pp. 80-84.

STEWART, J. (1982b). Two aspects of meaningful problem solving in science. Science Education, Vol. 66, pp. 734-749.
STEWART, J. (1983), Student problem solving in high school genetics. Science Education, Vol. 67, pp. 523-540.

STEWART, J. (1988). Potential leaming outcomes from solving genetics problems: A typology of problems. Science Education, Vol. $72(2)$, pp. $237-254$

STEWART, J. y HAFNER, R. (1991). Extending the conception of uproblem» in problem-solving research. Science Education, Vol. 75 , pp. 105-120.

STEWART, J., HAFNER, R. y DALE, M. (1990). Students' alternate views of meiosis. The American Biology Teacher, Vol. 52 (4), pp. 228-232.

STEWART, J. y KURK, J.V. (1990). Understanding and problemsolving in classical genetics. International Journal of Science Education, Vol. 12 (5), pp. 575-588.

THOMSON, N. y STEWART, J. (1985). Secondary school genetics instruction: making problem solving explicit and meaningful. Journal of Biological Education, Vol. 19 (1), pp. 53-62.

TOLMAN, R.R. (1982). Difficulties in genetics problem solving. The American Biology Teacher, Vol. 44 (9), pp. 525-527.

WALKER, R.A., HENDRIX, J.R. y MERTENS, T.R. (1980). Sequenced instruction in genetics and Piagetian cognitive development. The American Biology Teacher, Vol. 42, pp. 104-108. 
